\title{
LA INTERPRETACIÓN CUALITATIVA DE LA FÍSICA MATEMÁTICA DE PLATÓN COMO FACTOR EN LAS LIMITACIONES DE SU DESARROLLO
}

\author{
THE QUALITATIVE INTERPRETATION OF PLATO'S MATHEMATICAL
} PHYSICS AS A FACTOR IN THE LIMITATIONS OF ITS DEVELOPMENT

\section{Pablo Melogno ${ }^{1}$ \\ Universidad de la República (Universidad de la República)}

Recibido: 15-10-2013

Aceptado: 14-02-2014

\begin{abstract}
Resumen: Se propone un análisis de la física del Timeo y su interpretación en el Epinomis, mostrando que éste cancela el tratamiento de problemas matemáticos identificados en el Timeo, postulando además la existencia del éter. Se defiende que estas divergencias responden a la influencia de Aristóteles, que dará lugar a una física cualitativa despojada de bases matemáticas. Se concluye que estos elementos son de relieve para dar cuenta del limitado desarrollo de la física matemática de Platón, así como de la separación entre física y matemáticas operada en el pensamiento antiguo.
\end{abstract}

Palabras-clave: Platón, Timeo, Epinomis, Aristóteles, física, matemáticas.

[1] (pmelogno@gmail.com) Profesor de Filosofía por el Instituto de Profesores Artigas, Uruguay. Licenciado en Psicología por la Facultad de Psicología de la Universidad de la República (UdelaR), Uruguay. Master en Filosofía Contemporánea por la Facultad de Humanidades y Ciencias de la Comunicación (UdelaR). Profesor Adjunto de Epistemología e Historia de la Ciencia en el Instituto de Información de la Facultad de Información y Comunicación de la UdelaR. Ha realizado estancias de investigación en la Universidad Autónoma Metropolitana (México), y la Universidad de Buenos Aires (Argentina). Ha publicado diversos artículos en temas de filosofía e historia de la ciencia, en revistas y volúmenes colectivos de Uruguay, Venezuela, Argentina, Perú, España y México. Es compilador de Elementos de Historia de la Ciencia (CSE, 2011), Actas del $1^{\circ}$ Congreso de la Sociedad Filosófica del Uruguay (SFU, 2012), Problemas en Filosofía de la Ciencia (UdelaR, 2013) y Desafíos de la Educación Científica (UdelaR, 2013). 


\begin{abstract}
The following paper introduces an analysis of the physics in the Timaeus and its interpretation in the Epinomis, which will show that the latter cancels the treatment of mathematical problems appearing in the Timaeus, and, at the same time, it also postulates the existence of the ether. It is considered that these divergences respond to the influence of Aristotle, which will give rise to a qualitative physics deprived form mathematical basis. In the light of this, it is concluded that the previous elements are significant to explain the limited development of the mathematical physics in Plato as well as to cast light on the distinction between physics and mathematics belonging to the ancient thought.
\end{abstract}

Key-words: Plato, Timaeus, Epinomis, Aristotle, physics, mathematics.

\title{
1. El dodecaedro en la física de Platón ${ }^{2}$
}

En el diálogo Timeo, escrito aproximadamente en el 360 a.C. ${ }^{3}$, Platón expone un amplio sistema cosmológico, que integra problemas como el origen del universo, las regularidades matemáticas de las órbitas planetarias, la estructura de la materia y las transformaciones de los elementos físicos ${ }^{4}$. En los problemas vinculados a la composición del mundo físico, el diálogo sigue la tendencia predominante en el pensamiento griego, asumiendo que la diversidad de los elementos puede reducirse a tierra, fuego, agua y aire ${ }^{5}$. Sin embargo, resulta novedosa la estrategia de reconstrucción geométrica del mundo material, que intenta dar cuenta de las relaciones entre los cuatro elementos básicos a través de una serie de conceptos matemáticos. En esta medida, el Timeo incluye un programa de explicación matemática de los elementos físicos, tanto como un programa de explicación matemática de los movimientos planetarios ${ }^{6}$. El primero tiene como elementos centrales la aplicación de los cinco poliedros regulares a la física, y la identificación de cuatro de ellos con la base matemá-

[2] El autor quisiera agradecer a los Profs. Marina Camejo (UdelaR, Uruguay), y Godfrey Guillaumin (UAM, México), por las sugerencias sobre el presente trabajo.

[3] Para una discusión sobre la fecha del diálogo, Cherniss, Harold, The Relation of the Timaeus to Plato's Later Dialogues, The American Journal of Philology, 78 (3), 1957, pp. 225-266.

[4] Los comentarios clásicos del Timeo son el de Taylor, Alfred, A Commentary on Plato's Timaeus, Oxford, The Clarendon Press, 1928, y el de Cornford, Francis. Plato's Cosmology: the Timaeus of Plato London, Routledge and Kegan Paul, 1937. La edición crítica más reciente es la de Donald J. Zeyl, Timaeus, Indianapolis, Hackett Publishing Co., 2000.

[5] Se encuentran exposiciones interesantes de la cosmología del Timeo en Reale, Giovanni, Por una nueva interpretación de Platón, Barcelona, Herder, 2003 (1997), y Johansen, Thomas Kjeller, The Timaeus and The Principles of Cosmology. En G. Fine (ed.), The Oxford Handbook on Plato, Oxford, Oxford University Press, 2008.

[6] Sobre el status programático de la astronomía platónica, puede consultarse Mourelatos, Alexander, Astronomy and kinematics in Plato's project of rationalist explanation, Studies in History and Philosophy of Science, 12A (1), 1981, pp. 1-32.

THÉMATA. Revista de Filosofía, Nº49 enero-junio (2014) pp.: 217-234 doi: 10.12795/themata.2014.i49.12 
tica de los cuatro elementos ${ }^{7}$. El segundo se define por la imagen de un cosmos geocéntrico, finito y esférico, con órbitas planetarias circulares ordenadas conforme a progresiones matemáticas ${ }^{8}$.

El proyecto de matematización de la astronomía defendido por Platón presenta un amplio desarrollo en los siglos posteriores ${ }^{9}$, contribuyendo de forma decisiva a consolidar las relaciones entre matemáticas y astronomía. El programa físico por el contrario, no alcanza un nivel de desarrollo ni remotamente similar, quedando relegado en el marco de un paulatino proceso de disociación entre física y matemática, que tendrá su expresión más clara en la física de Aristóteles. En vistas de esto, el estudio de los problemas que deja abiertos la física matemática del Timeo, así como de la forma en que estos fueron abordados por los sucesores inmediatos de Platón, es clave para dar cuenta de cómo el platonismo da lugar a un programa matemático sumamente exitoso en astronomía y a otro más bien fallido en física. A su vez, el escaso desarrollo de la física matemática del Timeo es de relieve para dar cuenta de las condiciones históricas y conceptuales bajo las que la ciencia antigua desarrolló una tradición astronómica claramente matemática y una tradición física mayoritariamente no-matemática.

En principio, la física de Platón responde a los términos generales de la tradición pitagórica, que implicaban la creencia en el carácter divino de los astros y del mundo celeste en general, con la consiguiente imposibilidad de reducir ambos planos -terrestre y celeste- a los mismos principios explicativos. Es por esto que en la física platónica el dominio de aplicación de los principios asociados a los poliedros regulares se restringe a los cuatro elementos terrestres, contando para la astronomía y para el estudio de los cuerpos celestes con otra serie de principios matemáticos independientes. Para efectuar en el ámbito de la física esta transición desde los elementos materiales hasta sus bases matemáticas, el Timeo parte de que la materia posee volumen, y a su vez todo

[7] Tim. 53c-57b. Todas las referencias corresponden a la edición a cargo de R. G. Bury, Plato, Timaeus, London, The Loeb Classical Library, Harvard University Press/ William Heinemann, 1952.

[8] Ibid, 32c-40c. Exposiciones clásicas de la astronomía de Platón se encuentran en Heat, Thomas, History of Greek Mathematics, Oxford, Clarendon Press, 1921, v. I, y Hanson, Norwood Russell, Constelaciones y conjeturas, Madrid, Alianza, 1978 (1973). Para una discusión del status de la astronomía en Platón y su relación con la observación, puede verse Vlastos, Gregory, The role of observation in Plato's conception of astronomy. En Anton, J. P. (ed.), Science and the Sciences in Plato, Delmar-New York, Caravan Books, 1980.También Bulmer-Thomas, Ivor, Plato's Astronomy, The Classical Quarterly, New Series, 34 (1), 1984, pp. 107-112, y Gregory, Andrew, Astronomy and Observation in Plato's Republic, Studies in History and Philosophy of Science, 27 (4), 1996, pp. 451-471.

[9] Un raconto del debate sobre el impacto de Platón en la historia de la astronomía y en general de la ciencia, puede encontrarse en Lloyd, G. E. R., Plato as a Natural Scientist, The Journal of Hellenic Studies, 88, 1968, pp. 78-92. Una valoración sumamente crítica de la influencia de Platón aparece en Zhmud, Leonid, Plato as 'Architect of Science', Phronesis, 43 (3), 1998, pp. 211-244.

THÉMATA. Revista de Filosofía, Nº 49 enero-junio (2014) pp.: 217-234

doi: 10.12795/themata.2014.i49.12 
lo que posee volumen también tiene superficie; por lo que todos los cuerpos constan de superficies que conforman volúmenes ${ }^{10}$. A su vez, toda superficie puede ser dividida en triángulos, y todos los triángulos pueden ser seccionados hasta reducirlos a dos: el rectángulo isósceles y el rectángulo escaleno; por lo que éstos dos triángulos serían los componentes básicos e irreductibles -stoicheia- del mundo físico ${ }^{11}$.

En una posición intermedia entre los elementos materiales y los dos triángulos básicos, Platón introduce los cinco poliedros regulares, partiendo de que si los objetos materiales tridimensionales están compuestos de triángulos, debe haber algún tipo de entidad que sea tridimensional y al mismo tiempo reductible a triángulos. Esta doble condición se cumple en los poliedros regulares, sólidos cuyas caras están compuestas de planos equiláteros; el tetraedro, -4 caras triangulares- el cubo -6 caras cuadradas-, el icosaedro -20 caras triangulares-, el octaedro -8 caras triangulares-, y el dodecaedro -12 caras pentagonales- ${ }^{12}$. Los poliedros ofician como corpúsculos tridimensionales mínimos que componen los elementos, de modo que las diferencias en la composición de los poliedros explican la diversidad del mundo material.

Los corpúsculos cúbicos forman la tierra, ya que el cubo se compone de triángulos isósceles, distintos a los escalenos de los demás poliedros, y esto explica por qué la tierra no puede transformarse en ninguno de los otros tres elementos. Además, la tierra es el más sólido de los cuatro cuerpos, y el cubo es el que tiene la base más estable entre los poliedros; de esta forma queda el cubo identificado como sustrato de la tierra ${ }^{13}$. Para los elementos restantes, cuanto menor número de bases tiene un poliedro, mayor movilidad posee; por lo que al elemento más volátil le corresponderá el poliedro con menor número de caras. El fuego queda así identificado con el poliedro de menos caras, el tetraedro (4). $\mathrm{Al}$ agua, considerada como elemento menos móvil, le corresponde el poliedro de mayor número de caras, el icosaedro (20), y en un lugar intermedio se sitúa el aire, identificándose con el octaedro $\left(8\right.$ caras ${ }^{14}$.

Las caras del tetraedro (4), el octaedro (8), y el icosaedro (20), pueden reducirse a triángulos, seccionando a la mitad el triángulo equilátero que compone cada cara, de lo que resultan 2 triángulos rectángulos escalenos. Luego se traza una línea desde el vértice formado por la hipotenusa con el cateto menor de uno de los escalenos hasta la hipotenusa del otro -y viceversa-, obteniendo así 6 rectángulos escalenos. En el tetraedro, esta división arroja 6 triángulos escalenos por cada una de las 4 caras, por lo que la estructura básica del tetrae-

[10] Tim. 53c.

[11] Ibid, 53d-54b.

[12] Ibid, 54d-55c.

[13] Ibid, 55d-56a.

[14] Ibid, 56a-c.

THÉMATA. Revista de Filosofía, No49 enero-junio (2014) pp.: 217-234

doi: 10.12795/themata.2014.i49.12 
dro, y por tanto del fuego, estaría compuesta por 24 triángulos. En el octaedro, se obtienen 6 triángulos escalenos por cada una de las 8 caras, por lo que la estructura básica del octaedro y del aire es de 48 triángulos. Asimismo en el icosaedro se obtienen 6 triángulos escalenos por cada una de las 20 caras, lo que arroja una estructura del icosaedro y del agua consistente en 120 triángulos. Finalmente en el cubo, trazando las dos diagonales de los cuadrados que conforman cada cara se obtienen 4 triángulos rectángulos isósceles por cada una de las 6 caras, con una estructura de 24 triángulos como base del cubo y de la tierra.

Desde el momento en que el agua, el aire y el fuego están compuestos por triángulos rectángulos escalenos, es posible que cada uno de estos elementos se convierta en alguno de los restantes. El pasaje de un elemento a otro se produce por una redistribución de los triángulos que conforman los elementos involucrados en el proceso. Esto vale para aire, fuego, agua y sus respectivos poliedros, pero no para el cubo, ya que los triángulos rectángulos isósceles que lo componen no pueden formar ninguno de los otros tres poliedros, por esto es imposible que la tierra pueda transformarse en alguno de los otros tres elementos ${ }^{15}$.

Finalmente, la correlación de poliedros con elementos excluye el dodecaedro, al que Platón no identifica con ningún elemento ni le asigna procedimiento de triangulación alguno, atribuyéndole de modo muy poco claro la función de ser el modelo a partir de cual el Demiurgo diseña -diazographon- el $\operatorname{cosmos}^{16}$. La ambigüedad y brevedad de este pasaje, sumada a cierta asimetría que da como resultado el alinear cinco sólidos con cuatro elementos, generó desde la antigüedad numerosas interpretaciones de la función del quinto elemento en el Timeo, que bajo distintas perspectivas buscarán resolver los problemas que Platón dejó abiertos. En principio, pueden distinguirse en éstas dos vertientes incompatibles. Por un lado, la tendencia a identificar el dodecaedro con el quinto elemento o éter, que implica una cierta toma de distancia de la tesis del quinto poliedro como modelo del universo, al tiempo que permite completar la analogía entre cinco sólidos y cinco elementos ${ }^{17}$. En contraposición, se perfila la tendencia a asociar al dodecaedro con la esfera o con la estructura general del cosmos, en una tesitura más apegada al sentido literal del Timeo ${ }^{18}$.

\section{[15] Ibid, 56d-57b.}

[16] Ibid, 55c.

[17] La identificación del dodecaedro con el éter aparece en el diálogo Epinomis de Filipo de Opunte, así como también en los académicos Espeusipo y Jenócrates. El tratamiento del dodecaedro por parte de los discípulos inmediatos de Platón se revisa en Dillon, John, The Heirs of Plato: A Study of the Old Academy (347-274 BC), Oxford, Oxford University Press, 2003.

[18] Plutarco vinculó al dodecaedro con el zodíaco y con la esfera, emprendiendo la división de sus caras en triángulos bajo un procedimiento análogo -aunque no asimilable- al que propone el Timeo en los primeros cuatro poliedros. Plutarch, Platonic Questions. En Plutarch's Morals, Cambridge,

THÉMATA. Revista de Filosofía, No 49 enero-junio (2014) pp.: 217-234

doi: 10.12795/themata.2014.i49.12 
Nos proponemos aquí considerar un caso de esta primera línea interpretativa, que comienza a desarrollarse a muy poco de la muerte de Platón, a través del diálogo Epinomis del académico Filipo de Opunte. Pretendemos defender que el análisis del Epinomis muestra como ya en las primeras interpretaciones del Timeo surgidas dentro de la Academia, el programa matemático de Platón se vio interferido por elementos conceptuales ciertamente ajenos al platonismo. El origen de éstos puede ubicarse en la crítica a la teoría de las ideas efectuada por Aristóteles, que tiene un impacto específico en el Epinomis, tanto en la introducción de la hipótesis del éter como en la definida separación entre física y matemáticas efectuada en el diálogo. La consideración de estos procesos históricos e interpretativos resulta de relieve para la reconstrucción del trayecto histórico de la física matemática de Platón, a efectos de comprender por qué este programa no alcanza el mismo nivel de fertilidad y desarrollo heurístico logrado por la física de Aristóteles, o incluso por la misma astronomía matemática esbozada en el Timeo.

\section{El Epinomis y el éter}

Poco tiempo después de su aparición, el Timeo comienza a ser objeto de diferentes aproximaciones críticas, que se prolongarán a lo largo de la antigüedad. Una de las primeras se introduce en el diálogo apócrifo Epinomis, elaborado seguramente en el contexto de la Academia alrededor del 347-346 a.C. Antiguamente fue atribuido a Platón, no obstante hoy en día existe consenso respecto a que su autor fue el académico Filipo de Opunte, listado por Diógenes Laercio como discípulo directo de Platón, quien habría escrito la obra como un epílogo a las Leyes ${ }^{19}$. Glen Morrow ${ }^{20}$ señala que puede concederse tanto la autoría del diálogo por Filipo de Opunte como su identificación con el Filipo de Mende que Proclo -en el comentario a los Elementos- menciona dentro de los matemáticos antecesores de Euclides. Por su parte L. Zhmud ${ }^{21}$ considera a Filipo como el autor más probable de un fragmento incluido en la Historia de la

Press Of John Wilson and Son, 1874. Ed. William W. Goodwin. Esta estrategia también es ensayada por el platónico Alcino, según da noticia Heat, Ibid, I, pp. 296-297. Los intentos de seguir para el dodecaedro un proceso de triangulación similar al de los restantes poliedros dan cuenta de un tratamiento estrictamente matemático de los problemas de la física platónica, que mantiene -aunque no siempre con firmeza- lazos conceptuales definidos entre física y matemáticas. Una reconstrucción de los mecanismos de inscripción de los cinco los poliedros en la esfera, con énfasis en la función del dodecaedro, es ofrecida por Kotrč, R. F., The Dodecahedron in Plato's 'Timaeus', Rheinisches Museum für Philologie, 124 (3-4), 1981, pp. 212-222.

[19] Hicks, Robert, Drew, Diogenes Laertius: Lives of Eminent Philosophers, London, Harvard University Press/ William Heinemann, 1965.

[20] Morrow, Glenn R., Plato and the Mathematicians: An Interpretation of Socrates' Dream in the Theaetetus (201e- 206c), The Philosophical Review, 79 (3), 1970, pp. 309-333.

[21] Ibid, pp. 219-221, 238.

THÉMATA. Revista de Filosofía, Nº49 enero-junio (2014) pp.: 217-234

doi: 10.12795/themata.2014.i49.12 
Academia de Filodemo, y de una serie de tratados astronómicos y matemáticos que se le atribuyen en la Enciclopedia Suidas.

El Epinomis tiene como objetivo responder a la pregunta acerca de qué es la sabiduría -phronēsis-, y qué tipo de razonamiento dispone a la naturaleza humana al saber ${ }^{22}$. Esta función es otorgada a la matemática, considerada como la ciencia que hace sabios a los hombres. La reivindicación del conocimiento matemático se procesa en estrecha ligazón con la astronomía y con la teología astral de oriente, en cuanto los números se consideran un don divino concedido al hombre por la máxima divinidad, el universo -ouranós-, para comprender las revoluciones de los cuerpos celestes ${ }^{23}$. En lo que hace a la astronomía, el diálogo sigue a grandes rasgos las tesis del Timeo, y en el tratamiento de la naturaleza de los planetas se limita a reproducir las formulaciones platónicas originales sin introducir variaciones de relieve ${ }^{24}$, y sin siquiera mencionar las progresiones introducidas por Platón para dar cuenta de las distancias planetarias ${ }^{25}$.

En el campo de la física, el Epinomis incluye un esquema de la composición cualitativa del cosmos, aunque severamente despojado de los principios matemáticos atribuidos por Platón a los elementos. Aparecen en primer término los seres terrestres, mayormente compuestos de tierra. En contraste se ubica la especie de los astros, de carácter divino, mayormente constituida por fuego, aunque no sin partes de tierra y aire ${ }^{26}$. Se incluyen luego tres especies de seres intermedios, conforme a la división original de los elementos. Las primeras dos, éter y aire corresponden a dos especies distintas de espíritus divinos -daemon-, mediadores entre los dioses y los hombres; la tercera es la especie de los seres de agua, semidioses respecto de los que no se abunda en detalles ${ }^{27}$.

En este marco, el dodecaedro es asociado con el éter ${ }^{28}$, quinto elemento ubicado de modo algo impreciso en el espacio comprendido entre la esfera de las estrellas fijas y la Tierra, y vinculado asimismo a la demonología 29. La idea del éter como sustancia diferenciada en principio es ajena a la obra de Platón, en la que apenas aparece como una variedad del aire ${ }^{30}$. Sólo se

[22] Epin. 973a. Todas las referencias corresponden a la edición a cargo de W. R. M. Lamb, Plato, Epinomis, London, The Loeb Classical Library, Harvard University Press/ William Heinemann, 1950.

[23] Ibid, 975b-976d.

[24] Algunos problemas matemáticos del Timeo vinculados con las órbitas planetarias, se tratan en Van Der Waerden, B. L., The motion of Venus, Mercury and the Sun in early greek astronomy, Archive for History of Exact Science, 26 (2), 1982, pp. 99-113.

[25] Tim. 35b-37c.

[26] Epin. 981c-982a.

[27] Ibid, 984e-985b.

[28] Ibid, 1982a.

[29] Ibid, 981c, 983d, 985a.

[30] Tim. 58d. Cratilo 408d. Las referencias a las restantes obras de Platón corresponden a:

THÉMATA. Revista de Filosofía, No 49 enero-junio (2014) pp.: 217-234

doi: 10.12795/themata.2014.i49.12 
considera como sustancia celeste en el Fedón ${ }^{31}$, en un contexto polémico de discusión de la cosmología de los filósofos materialistas. En el Epinomis sin embargo, el éter aparece como un quinto elemento diferenciado de pleno derecho, y su identificación con el dodecaedro se procesa en un plano de consideración estrictamente cualitativa de los problemas de la composición de la materia. Esto hace que en la introducción del quinto poliedro no se trate ninguna de las consecuencias matemáticas que puede reportar su identificación con el éter, ni otros problemas matemáticos de orden más general, como la triangulación de sus caras o la relación de los triángulos fundamentales del dodecaedro con los restantes poliedros ${ }^{32}$. Por otra parte, una vez que el dodecaedro es postulado como sustrato de un elemento específico, queda cancelada la posibilidad de considerarlo como modelo del universo, alternativa mencionada en el Timeo y omitida en el Epinomis.

De esta manera, al mismo tiempo que el Epinomis aparece como una apología del conocimiento matemático, restringe fuertemente la aplicación de la matemática a la astronomía, bajo la premisa de que sólo los objetos celestes son dignos del conocimiento matemático, por tener carácter divino. Por contrapartida, las especies terrestres son excluidas del campo de aplicación de la matemática, y esto provoca que los problemas de la física matemática del Timeo aparezcan actualizados en un tratamiento cualitativo que discontinúa una de las principales orientaciones heurísticas de la física matemática de Platón. El carácter cualitativo del Epinomis está dado por el hecho de que las diferencias entre los elementos son explicadas por las diferencias entre las cualidades que definen a cada elemento, sin que haya sin que haya una base matemática que permita dar cuenta de dichas cualidades. El Timeo por el contrario involucra una perspectiva cuantitativa, ya que si bien no supone procedimientos de medición o control empírico asociados a la matematización de un dominio de fenómenos -como sí será el caso de la astronomía griega-, involucra una serie de principios que suponen una cuantificación reconstructiva de la materia, a partir de asignar a cada elementos un número determinado de triángulos.

Así, el Epinomis no hace otra cosa que suprimir lo que G. E. R. Lloyd denominó la tentativa platónica de especificar las aplicaciones posibles de la razón teórica en el mundo del devenir ${ }^{33}$, mediante la restricción del dominio de aplicación de la matemática a la astronomía. A este respecto, Cornford postuló

Platón, Obras Completas, Madrid, Aguilar, 1972.

[31] 109d-112a.

[32] En una reconstrucción actual de algunos de estos problemas, Visintainer ha defendido que la exclusión del dodecaedro responde a que en el esquema del Timeo es matemáticamente imposible incluir más elementos que los correspondientes a los cuatro primeros poliedros. Visintainer, John, A Potential Infinity of Triangle Types. On the Chemistry of Plato's Timaeus, International Journal for Philosophy of Chemistry, 4 (2), 1998, pp. 117-128.

[33] Ibid, p. 82.

THÉMATA. Revista de Filosofía, Nº49 enero-junio (2014) pp.: 217-234 doi: 10.12795/themata.2014.i49.12 
que en el Epinomis se opera una reducción de todas las ciencias a su expresión numérica, en la medida en que las matemáticas aparecen como el fundamento de las disciplinas particulares ${ }^{34}$. Sin embargo, es necesario insistir en que esta reducción matemática excluye a la física, derivando en un proyecto de matematización de las ciencias de carácter parcial y restringido. En consonancia con el Platón de la República, el Epinomis supone que los cuerpos terrestres presentan un movimiento desordenado, carente de regularidad y armonía, insusceptible por tanto de ser tratado matemáticamente ${ }^{35}$. Al respecto Johan$\operatorname{sen}^{36}$ señala que la aplicación de la matemática en el Timeo representa una ampliación de su campo y de sus posibilidades de conocimiento, ampliación que es suprimida en el Epinomis al asociar el dodecaedro con el éter bajo una perspectiva cualitativa y desmatematizada. Así, por más que el Epinomis se mantiene fiel al Timeo en la asociación de elementos materiales con sólidos matemáticos, se desprende de éste al defender que el estudio de la materia no es pasible de tratamiento matemático.

\section{La influencia aristotélica}

La introducción del éter y su identificación con el dodecaedro, así como la supresión de los problemas matemáticos asociados a la física de los poliedros, constituyen elementos de ruptura del Epinomis respecto del Timeo. Puede afirmarse que este distanciamiento del proyecto platónico de una física matemática responde a la influencia tempranamente ejercida por las ideas de Aristóteles sobre Filipo de Opunte, que terminará configurando en el Epinomis una suerte de lectura aristotélica de la física de Platón. La elucidación de este punto involucra por lo menos dos componentes, uno estrictamente cronológico, relativo al grado de desarrollo intelectual y producción que había alcanzado Aristóteles al momento de la elaboración del diálogo de Filipo, y otro de índole conceptual, que remite a establecer con precisión cuáles son los indicadores de esta incipiente influencia aristotélica.

A principios del siglo XX, los ahora clásicos estudios de Werner Jaeger mostraron que la oposición de Aristóteles a Platón no es expresión sistemática de la época de madurez intelectual de aquél, sino que responde a un proceso de distanciamiento gradual respecto del platonismo, que se remonta a los últimos años en que Aristóteles permaneció en la Academia. Jaeger identificó así un Aristóteles platónico ${ }^{37}$, que toma distancia de ciertas tesis específicas de su

[34] Cornford, Francis, Mathematics and Dialectic in the Republic VI.-VII. (II.), Mind, New Series, 41 (162), 1932, p. 180.

[35] Rep. 978a-b, 982a-b.

[36] Ibid.

[37] Cuyas expresiones ubica en los libros más antiguos de la Metafísica y en la Física, así como

THÉMATA. Revista de Filosofía, Nº49 enero-junio (2014) pp.: 217-234

doi: 10.12795/themata.2014.i49.12 
maestro pero manteniendo algunas premisas generales de la escuela. En este contexto de revisión temprana del platonismo, localiza Jaeger la introducción de la hipótesis del éter; y apelando a Cicerón ${ }^{38}$ entre otras fuentes, concluye que Aristóteles tiene que haber propuesto la hipótesis mientras estaba en la Academia, más específicamente en el diálogo perdido De la Filosofía, escrito probablemente entre el 348-34739. Ahora bien, el Epinomis habría sido escrito o bien al mismo tiempo que De la Filosofía ${ }^{40}$, o bien en un tiempo inmediatamente posterior ${ }^{41}$. En uno u otro caso, la hipótesis del éter es rápidamente incorporada en el Epinomis, tomando la expresión quinto cuerpo del tratado aristotélico, del que también son extraídas en términos casi literales las consideraciones de Aristóteles sobre el carácter divino de los cuerpos celestes y del universo mismo $\mathrm{o}^{42}$. La rápida incorporación de la novedosa hipótesis aristotélica del éter, en el marco de una emergente concepción cualitativa de la física y de la naturaleza de los elementos ${ }^{43}$, puede considerarse como resultado directo de la orientación que estaban tomando las discusiones cosmológicas en la Academia, y del escaso impacto que el proyecto físico-matemático de Platón había tenido entre algunos de sus discípulos ${ }^{44}$.

Buscando dar cuenta de esta irrupción de elementos aristotélicos en el seno del platonismo, Niehoff ha señalado recientemente ${ }^{45}$ que ya desde los últimos días de Platón en la Academia, comienza a forjarse un fuerte acuerdo en las lecturas del Timeo efectuadas por platónicos y posteriormente por aris-

en los tratados perdidos De la Filosofía y Protréptico. Jaeger, Werner, Aristóteles, México, FCE, 1995 (1923).

[38] Cicero, De natura deorum, London, The Loeb Classical Library, Harvard University Press/ William Heinemann, 1967. Ed. H. Rackham.

[39] Jaeger, ibid, pp. 162-163. Sobre la participación de Aristóteles por esta época en los debates de la Academia, puede consultarse Méndez Lloret, Isabel, La concepción aristotélica de la divinidad: del Perì philosophías a Metafísica XII; teología cósmica y motores inmóviles, Daímwn. Revista de Filosofía, 6, 1993, pp. 23-40. Para un tratamiento crítico de la introducción del éter en De la Filosofía, es de relieve el trabajo de Berti, Enrico, La filosofia del primo Aristotele, Padova, CEDAM, 1997 (1962),

[40] Aubenque, Pierre, El problema del Ser en Aristóteles, Madrid, Taurus, 1981 (1962), pp. 323-325.

[41] Jaeger, ibid, p. 175. Berti, ibid, p. 326 y ss.

[42] Jaeger, ibid, p. 163.

[43] Johansen, Thomas, From Plato's Timaeus to Aristotle's De Caelo: The Case of the Missing World Soul. En Wildberg, C. and Bowen, A.C. (eds.), New Perspectives on Aristotle's De Caelo, Leiden/Boston, Brill, 2009, 9-28.

[44] Jaeger, insiste en que Aristóteles no sólo coincidió con Filipo de Opunte en la Academia, sino que discutió directamente con él, ibid, p. 129. Una perspectiva interesante de las relaciones entre las obra de ambos se encuentra en Falcon, Andrea, Aristotle \& the Science of Nature, CambridgeUK, Cambridge University Press, 2005, p. 77 y ss.

[45] Niehoff, M. R., Did the Timaeus Create a Textual Community?, Greek, Roman, and Byzantine Studies, 47, 2007, pp. 164-165.

THÉMATA. Revista de Filosofía, No 49 enero-junio (2014) pp.: 217-234 doi: 10.12795/themata.2014.i49.12 
totélicos, de lo que es indicador la tendencia a interpretar metafóricamente la creación del cosmos por el demiurgo platónico ${ }^{46}$, haciéndola compatible con la afirmación de la eternidad del cosmos por parte de Aristóteles ${ }^{47}$. Esta conjunción de elementos platónicos y aristotélicos en la interpretación del Timeo es prolongada por Niehoff hasta Espeusipo, Jenócrates y Crantor de Soli, discípulos directos de Platón coincidentes con Filipo y Aristóteles en la Academia. Jaeger llegó aún más lejos en este punto, afirmando que las semejanzas entre Filipo y Aristóteles permiten inferir que ambos están exponiendo las doctrinas dominantes en la Academia ${ }^{48}$, cuya formación es resultado de un distanciamiento crítico respecto a algunas enseñanzas originales de Platón.

Hasta aquí puede colegirse que la influencia aristotélica en el Epinomis remite a un período en que Aristóteles ha introducido la hipótesis de éter, y ha tomando suficiente distancia de Platón como para renegar de las formulaciones básicas del platonismo, lo que resultará de relieve para el abandono de la física matemática del Timeo. Queda cierto margen para cuestionar si se trata literalmente de una influencia de Aristóteles en el autor del Epinomis, o si por el contrario, el Epinomis y De la Filosofía deben ser considerados equitativamente como expresiones de las mutuas influencias ejercidas entre los académicos, y de la dirección que había tomado el debate cosmológico en la Academia. Se trata de una cuestión antes histórica que conceptual, y no parece fácil de resolver en vistas del exiguo conocimiento que tenemos de la figura de Filipo de Opunte. Sin embargo, desde el punto de vista de la reconstrucción conceptual, puede sí afirmarse que la perspectiva cualitativa de consideración del mundo físico que asoma en el Epinomis ya venía siendo desarrollada por Aristóteles, con quien posteriormente alcanzará su expresión más acabada.

Inicialmente, Aristóteles tomaba al éter como principio de vida y movimiento de los astros, en analogía a la forma en que los restantes elementos son principio de vida y movimiento de los objetos terrestres ${ }^{49}$. Posteriormente en Meteorología ${ }^{50}$ los antecedentes del éter serán situados en la tradición jonia y especialmente en Anaxágoras, lo que da cuenta de la pervivencia de las tradiciones materialistas en la crítica aristotélica a Platón ${ }^{51}$. A su vez, la introducción del éter es contemporánea a las primeras críticas de Aristóteles a la teoría

[46] Tim. 28b-c.

[47] Fis. VIII, 256a-261b. Metaf. XII, 1072a-1073a. Ciel. I, 279b-284a. Todas las referencias a Aristóteles, salvo indicación, corresponden a Aristóteles, Obras, Madrid, Aguilar, 1973.

[48] Jaeger, ibid, p. 176, 354.

[49] Jaeger, ibid, pp. 167-168.

[50] Meteor. I, 339a, II, 365a. Aristotle, Meteorologica, London, The Loeb Classical Library, Harvard University Press/ William Heinemann, 1952. Ed. H. D. P. Lee.

[51] Quien había hecho una mención lateral del éter justamente en una discusión de la cosmología de los filósofos materialistas. Fedón. 109d-112a.

THÉMATA. Revista de Filosofía, Nº 49 enero-junio (2014) pp.: 217-234

doi: 10.12795/themata.2014.i49.12 
de las ideas expuestas en el capítulo 9 de libro I de la Metafísica ${ }^{52}$. A partir de estas fases tempranas de su desarrollo intelectual, la ruptura de Aristóteles con la teoría de las ideas terminará involucrando aspectos fundamentales del platonismo, cuyo cuestionamiento derivará en una reorganización de las disciplinas de conocimiento, que tendrá entre sus consecuencias principales la separación entre física y matemáticas y el desarrollo de una física cualitativa.

En este marco, la crítica de Aristóteles a Platón y a los platónicos involucra por lo menos la negación de cuatro tesis: 1. la existencia de las ideas como sustancias -ousíai-independientes; 2 la existencia de los números como sustancias independientes; 3. la identificación de los números con las ideas; y finalmente 4 . la posibilidad de que tanto ideas como números puedan ser principios o elementos constitutivos de la materia ${ }^{53}$. El desarrollo intelectual de Aristóteles lo llevó ya desde épocas tempranas en la Academia a negar la sustancialidad de los números, así como de puntos, líneas, figuras y cuerpos geométricos tridimensionales ${ }^{54}$, y una vez que el Timeo supone el carácter sustancial de los poliedros que dan base a los elementos ${ }^{55}$, el proyecto platónico de matematización del mundo físico quedó comprometido por la crítica a las ideas. Finalmente, ello tendrá como resultado la negación específica de la tesis de la reducción de elementos a poliedros, efectuada en De la generación y corrupción $^{56}$ y Del Cielo ${ }^{57}$.

Bajo este cuestionamiento al status ontológico de ideas y números, Aristóteles dará forma a un sistema físico de carácter cualitativo, ya que el integrar la física con la matemática conllevaba de forma inevitable el riesgo de la reificación de los números operada por los pitagóricos y por Platón. El desarrollo de una física no matemática parece así ser una consecuencia de la negación del carácter sustancial de los objetos matemáticos, así como de la posibilidad de postularlos como sustrato del mundo físico. Estas premisas desarrolladas tempranamente por Aristóteles no se siguen literalmente en la física del Epinomis, en cuanto éste no niega la sustancialidad de los objetos matemáticos, sino que por el contrario, exalta su potencialidad epistémica y su carácter sagrado. Sin embargo, el Epinomis sí se ve permeado por el esquema aristotélico desde el momento en que excluye que los objetos matemáticos puedan oficiar como principios explicativos del mundo material. Así, sin encuadrarse literal-

[52] Jaeger, ibid, p. 201.

[53] Metaf. I, 990a-993a.

[54] Ibid, I, 992a-b.

[55] Crombie, I. M., Análisis de las doctrinas de Platón, Madrid, Alianza, 1963 (1988), p. 222. Lloyd, D. R., The Chemistry of Platonic Triangles: Problems in the Interpretation of the Timaeus, International Journal for Philosophy of Chemistry, 13 (2), 2007, p. 100.

[56] Gen. II, 328b-329a.

[57] Ciel. III, 306b-307b.

THÉMATA. Revista de Filosofía, Nº49 enero-junio (2014) pp.: 217-234 doi: 10.12795/themata.2014.i49.12 
mente en lo que con el tiempo será una feroz crítica de Aristóteles al realismo matemático, el Epinomis constituye un repliegue de la matemática respecto de la física, menos sistemático pero tan acusado como el que desarrollará el aristotelismo posterior.

Esto permite entrever que el distanciamiento entre física y matemáticas operado por los primeros platónicos responde a las limitaciones y cuestionamientos surgidos a la teoría de las ideas. Sin embargo, para el caso específico de Aristóteles, Jaeger parece haber dado más peso a las limitaciones técnicas como causa de la orientación cualitativa de la física: "carecía Aristóteles de los medios técnicos para hacer mediciones cuantitativas exactas, o determinar las condiciones cuantitativas de las cualidades, así que la investigación no podía avanzar en esa dirección." 58 Puesta la cuestión en estos términos, daría la impresión que en el marco conceptual de la física Aristóteles hubiera sido posible tanto una orientación cualitativa como una cuantitativa, y que lo segundo no fue posible por ausencia de dispositivos adecuados de medición. Cabe pensar sin embargo, que la carencia de medios técnicos no es causante de la orientación cualitativa de la física aristotélica, en cuanto esta tiene su origen en un marco conceptual que involucraba de forma casi inevitable el desarrollo de una física distanciada del espíritu matematizante de Platón. El que Aristóteles no haya orientado la investigación a la búsqueda de los medios necesarios para el tratamiento cuantitativo de las cualidades es resultado de decisiones filosóficas previas, vinculadas al abandono de algunos presupuestos centrales del platonismo. En este sentido, su creciente renuencia a aceptar la sustancialidad de ideas y números, parece haber precipitado la orientación cualitativa de su física, más allá de posibilidades técnicas y acceso a dispositivos de medición.

Por otra parte, tampoco Platón contaba con los medios técnicos necesarios para respaldar sus tesis sobre los poliedros, sin embargo ello no lo disuadió de defender el tratamiento matemático de los elementos materiales, en nombre de la asunción filosófica previa de que era posible construir una física de base matemática. Asimismo, ya en época de Aristóteles se registran algunos logros matemáticos significativos fuera del ámbito de la astronomía ${ }^{59}$; pero que no podían oficiar como un aliciente para que la física aristotélica adoptara una orientación cuantitativa - por mejores medios técnicos que dispusiera- ya que los términos en que Aristóteles rompe con el platonismo inclinan irreversible-

[58] Jaeger, ibid, p. 436.

[59] Izumi señala a este respecto que la reconstrucción matemática de los elementos emprendida en el Timeo se nutre directamente de los avances registrados por la estereometría en la época de Platón, lo cual refuerza la idea de que no fueron motivos relativos a los progresos registrados en cada disciplina los que movieron a Aristóteles a separar la física de la matemática, sino motivos de orden mucho más fundamental, vinculados a la naturaleza y estructura del conocimiento. Izumi, Chiye, Rethinking Timaeus 31b4-32c4: de facto Indestructibility of the Universe Founded on Stereometry, Journal of Classical Studies, 49, 2001, pp. 177-179.

THÉMATA. Revista de Filosofía, Nº49 enero-junio (2014) pp.: 217-234

doi: 10.12795/themata.2014.i49.12 
mente la balanza en favor de una perspectiva cualitativa, con la consiguiente disociación entre la matemática y el mundo empírico.

Esta impugnación de los principios fundamentales del platonismo tuvo impacto directo en el tratamiento que Aristóteles efectuó del Timeo, en cuanto una vez rechazada la existencia independiente -chorismós- de ideas y objetos matemáticos, no quedaba lugar para una física que pretendiera reducir las estructuras últimas de la materia a cuerpos geométricos. Si bien el Epinomis no acepta las premisas aristotélicas de cuestionamiento de la matemática, asume plenamente las consecuencias relativas a la desmatematización de la física. De este modo, el escaso desarrollo de la física matemática en el Epinomis responde a razones filosóficas de fondo, vinculadas a la naturaleza del conocimiento matemático y a su relación con el mundo físico ${ }^{60}$.

Por otra parte, Jaeger se apoya en las diferencias respecto al orden en que se disponen los elementos físicos, para señalar que el Epinomis incorpora la teoría aristotélica del éter de modo parcial y fallido, en una lectura adaptativa respecto del Timeo ${ }^{61}$. A este respecto puede concederse sin dificultad que en el Epinomis la hipótesis del éter no logra el grado de desarrollo y fecundidad que alcanzará en el corpus aristotélico, pero difícilmente esto pueda atribuirse a un intento de preservación del marco de Platón. Sólo podría hablarse de una adaptación de las nuevas ideas de Aristóteles al Timeo si los presupuestos básicos de la física de los poliedros permanecieran estables en el intento de ensamblarlos con la hipótesis del éter. Pero sucede justamente lo contrario, en la medida en que en el diálogo de Filipo es la perspectiva cualitativa de Aristóteles la que ingresa empobrecida pero sin distorsión, en una matriz platónica previa que se ve sometida a notorias alteraciones. En estos términos el Epinomis constituye más bien una estrategia de adaptación de la física de Platón a los nuevos conceptos de Aristóteles, en el transcurso de la cual quedan atrás importantes ingredientes del platonismo.

[60] Méndez Lloret, ibid, p. 25, llamó la atención sobre el silencio del Epinomis respecto de la teoría de las ideas, que es acompañado por la postulación del carácter divino del mundo celeste y de los astros. Señaló asimismo la relación de continuidad que se registra entre el Timeo, las Leyes, el Epinomis y el tratado De la Filosofía, respecto al status divino del mundo celeste y al desarrollo de la teología astral. Si bien pueden concederse de buen grado estas relaciones de continuidad en lo que hace a la estructura conceptual de la astronomía y sus implicancias teológicas, no puede tratarse en los mismos términos el tránsito de los últimos escritos de Platón al Epinomis y a los primeros tratados de Aristóteles en física y en astronomía. No sólo por las importantes diferencias entre la física aristotélica y la del Epinomis respecto del Timeo, sino también por el hecho de que Aristóteles no impugnó bajo ninguna forma el programa platónico de reconstrucción matemática de las órbitas planetarias, ni la tesis general de una ligazón estrecha entre astronomía y matemáticas -Metaf. XII, 1073b-, como sí lo hizo con la posibilidad de reconstruir matemáticamente la estructura de los elementos terrestres. Bajo esta perspectiva, no resulta problemático que la continuidad registrada en la astronomía y la teología astral sea simultánea a la discontinuidad registrada en el desarrollo de la física.

[61] Jaeger, ibid, p. 169.

THÉMATA. Revista de Filosofía, Nº49 enero-junio (2014) pp.: 217-234

doi: 10.12795/themata.2014.i49.12 
En tren de defender esta motivación platónica del Epinomis, afirma Jaeger "el único cambio hecho a la teoría de Platón es el de que en lugar del aire, el nivel más alto y más puro de lo que había llamado éter ya Platón mismo, tenemos dos distintos elementos. Así, el Epinomis, asimilando externamente la teoría del éter, elude con deliberación el elemento verdaderamente esencial de la idea." 62 . A este respecto puede concederse que el Epinomis representa una suerte de producto de transición entre la tradición de la Academia y las innovaciones del Aristóteles, pero difícilmente pueda pensarse este proceso en términos de conservación del platonismo ${ }^{63}$. El diálogo presenta la hipótesis del éter en términos bastante más fragmentarios de los que alcanzará con Aristóteles, porque lo decisivo del Epinomis en relación al Timeo no son los cambios que introduce por la positiva en el programa platónico, sino las orientaciones que cancela y los problemas cuya consideración omite. En este sentido el diálogo no implica una conservación de la física platónica, ya que la introducción de la hipótesis del éter es simultánea a la supresión de los problemas vinculados al sustrato matemático de los elementos y de la posibilidad misma de una física matemática.

En función de lo anterior, la influencia de Aristóteles en el Epinomis puede verse en dos aspectos, la introducción de la hipótesis del éter y la clausura del programa platónico de matematización de los elementos. El primero constituye una de las principales bases heurísticas del desarrollo posterior de la física aristotélica, que dará lugar a sendos desarrollos en la tradición posterior, relativos a la naturaleza del éter y su lugar en la cosmología ${ }^{64}$. El segundo es seguramente resultado de la renuncia de Aristóteles a la teoría de las ideas, así como a la alternativa de considerar a los números u objetos matemáticos como sustancias últimas, atribuida a Platón ${ }^{65}$ y desarrollada por los académicos Espeusipo y Jenócrates ${ }^{66}$. El rechazo de Aristóteles a la teoría de las ideas y a la reificación de los números lo condujo a una perspectiva fuertemente cualitativa de la naturaleza, en la que no sólo no cabía considerar a los nú-

[62] Ibid, pp. 168-169.

[63] En este sentido no resulta compartible la interpretación de Lacey, para quien en lo que hace a temas cosmológicos el Epinomis no sólo no aporta novedades de fondo, sino que trata de mantenerse estrictamente fiel al platonismo. Lacey, A. R., The Mathematical Passage in the Epinomis, Phronesis, 1 (2), 1956, p. 82. También Zhmud, ibid, p. 238, afirma que en el escrito de Filipo no hay ninguna idea astronómica que ya no esté contenida en los escritos de Platón. Sin embargo, en función de lo que hemos expuesto puede pensarse que estas lecturas del Epinomis como un producto conservador y apegado a la ortodoxia de la Academia, impiden ver las divergencias que presenta respecto del Timeo.

[64] Paiva de Andrade, Elaine, Faber, Jean, Pinguelli Rosa, Luiz, A Spontaneous Physics Philosophy on the Concept of Ether Throughout the History of Science: Birth, Death and Revival, Foundations of Science, 18 (3), 2013, pp. 559-577. Wildberg, C., John Philoponus Criticism of Aristotle's Theory of Aether, Berlin/ New York, de Gruyter, 1988.

[65] Metaf. I, 987b, 991b, XIII, 1083a, 1086a.

[66] Reale, ibid, caps. 2, 8.

THÉMATA. Revista de Filosofía, No 49 enero-junio (2014) pp.: 217-234

doi: 10.12795/themata.2014.i49.12 
meros como entidades con existencia independiente, sino que también dejaba cancelada la posibilidad de considerar a la matemática -siquiera a nivel metodológico- como una herramienta útil para la comprensión del mundo físico. Es así que en el Epinomis la física del Timeo es moldeada conforme a los términos de este emergente programa de tratamiento cualitativo del mundo físico y de restricción de la matemática al estudio de los cuerpos celestes.

\section{Conclusión}

La cosmología expuesta por Platón en el Timeo contiene dos programas relativamente independientes de aplicación de la matemática a otras disciplinas, que dan lugar respectivamente a una astronomía matemática y a una física matemática. El programa astronómico resulta sumamente fértil a lo largo de la historia, al contrario del programa físico, que durante la antigüedad registrará desarrollos solamente fragmentarios y parciales, no comparables al nivel de consolidación que alcanzará la física de Aristóteles. Las insuficiencias del proyecto platónico de matematización del mundo material se inscriben en un proceso central para la ciencia antigua, marcado por el desarrollo de una astronomía con un fuerte grado de matematización, y de una física cualitativa carente de bases matemáticas.

En este sentido, el Epinomis da cuenta de que ya en los primeros intentos de abordar los problemas planteados en el Timeo, su tratamiento aparece atravesado por elementos divergentes al platonismo, derivados de las discusiones cosmológicas dadas en la Academia. En el Epinomis, estas divergencias se trasuntan en la introducción de la hipótesis del éter, la supresión de los problemas físico-matemáticos abiertos en el Timeo, y la asunción de una física cualitativa que se distancia del proyecto platónico de matematización de los fenómenos materiales, confinando la aplicación de las matemáticas al campo de la astronomía.

La crítica efectuada por Aristóteles a la teoría de las ideas es por demás relevante para entender este proceso de ruptura de Filipo de Opunte con la física de Platón, particularmente en lo que hace a la negación de la existencia independiente de las ideas y de los objetos matemáticos, así como de la posibilidad de considerar a éstos como el sustrato último de la materia. Puede pensarse entonces que desde el punto de vista histórico, la física matemática del Timeo quedó incrustada entre las tradiciones materialistas previas y el incipiente desarrollo de la física aristotélica, lo cual es de importancia para entender el escaso impacto que tuvo, incluso entre los discípulos inmediatos de Platón. Por último, concediendo que la crítica de Aristóteles a la teoría de la ideas tuvo impacto directo en esta disociación entre física y matemáticas, puede señalarse que el desarrollo de una física cualitativa y la restricción de la matemática al campo astronómico, respondió originalmente a decisiones filosóficas de fondo, relativas a la naturaleza del conocimiento y la clase de entidades que es lícito postular para explicar los fenómenos naturales. 
La interpretación cualitativa de la física matemática de Platón como factor en las limitaciones de su desarrollo

\section{Referencias bibliográficas}

Aristóteles, Obras, Madrid, Aguilar, 1973.

Aristotle, Meteorologica, London, The Loeb Classical Library, Harvard University Press/ William Heinemann, 1952. Ed. H. D. P. Lee.

Aubenque, Pierre, El problema del Ser en Aristóteles, Madrid, Taurus, 1981 (1962), pp. 323-325.

Berti, Enrico, La filosofia del primo Aristotele, Padova, CEDAM, 1997 (1962),

Bulmer-Thomas, Ivor, Plato's Astronomy, The Classical Quarterly, New Series, 34 (1), 1984, pp. 107-112.

Cherniss, Harold, The Relation of the Timaeus to Plato's Later Dialogues, The American Journal of Philology, 78 (3), 1957, pp. 225-266.

Cicero, De natura deorum, London, The Loeb Classical Library, Harvard University Press/ William Heinemann, 1967. Ed. H. Rackham.

Cornford, Francis, Mathematics and Dialectic in the Republic VI.-VII. (II.), Mind, New Series, 41 (162), 1932, p. 180.

Cornford, Francis. Plato's Cosmology: the Timaeus of Plato London, Routledge and Kegan Paul, 1937.

Crombie, I. M., Análisis de las doctrinas de Platón, Madrid, Alianza, 1963 (1988).

Dillon, John, The Heirs of Plato: A Study of the Old Academy (347-274 BC), Oxford, Oxford University Press, 2003.

Falcon, Andrea, Aristotle \& the Science of Nature, Cambridge-UK, Cambridge University Press, 2005.

Gregory, Andrew, Astronomy and Observation in Plato's Republic, Studies in History and Philosophy of Science, 27 (4), 1996, pp. 451-471.

Hanson, Norwood Russell, Constelaciones y conjeturas, Madrid, Alianza, 1978 (1973). Heat, Thomas, History of Greek Mathematics, Oxford, Clarendon Press, 1921, v. I. Hicks, Robert, Drew, Diogenes Laertius: Lives of Eminent Philosophers, London, Harvard University Press/ William Heinemann, 1965.

Izumi, Chiye, Rethinking Timaeus 31b4-32c4: de facto Indestructibility of the Universe Founded on Stereometry, Journal of Classical Studies, 49, 2001, pp. 177-179.

Jaeger, Werner, Aristóteles, México, FCE, 1995 (1923).

Johansen, Thomas, The Timaeus and The Principles of Cosmology. En G. Fine (ed.), The Oxford Handbook on Plato, Oxford, Oxford University Press, 2008.

Johansen, Thomas, From Plato's Timaeus to Aristotle's De Caelo: The Case of the Missing World Soul. En Wildberg, C. and Bowen, A.C. (eds.), New Perspectives on Aristotle's De Caelo, Leiden/Boston, Brill, 2009, 9-28.

Kotrč, R. F., The Dodecahedron in Plato's 'Timaeus', Rheinisches Museum für Philologie, 124 (3-4), 1981, pp. 212-222.

Lacey, A. R., The Mathematical Passage in the Epinomis, Phronesis, 1 (2), 1956. Lloyd, D. R., The Chemistry of Platonic Triangles: Problems in the Interpretation of the Timaeus, International Journal for Philosophy of Chemistry, 13 (2), 2007.

THÉMATA. Revista de Filosofía, Nº49 enero-junio (2014) pp.: 217-234

doi: 10.12795/themata.2014.i49.12 
Lloyd, G. E. R., Plato as a Natural Scientist, The Journal of Hellenic Studies, 88, 1968, pp. 78-92.

Méndez Lloret, Isabel, La concepción aristotélica de la divinidad: del Perì philosophías a Metafísica XII; teología cósmica y motores inmóviles, Daímwn. Revista de Filosofía, 6, 1993, pp. 23-40.

Morrow, Glenn R., Plato and the Mathematicians: An Interpretation of Socrates' Dream in the Theaetetus (201e- 206c), The Philosophical Review, 79 (3), 1970, pp. 309-333.

Mourelatos, Alexander, Astronomy and kinematics in Plato's project of rationalist explanation, Studies in History and Philosophy of Science, 12A (1), 1981, pp. 1-32.

Niehoff, M. R., Did the Timaeus Create a Textual Community?, Greek, Roman, and Byzantine Studies, 47, 2007, pp. 164-165.

Paiva de Andrade, Elaine, Faber, Jean, Pinguelli Rosa, Luiz, A Spontaneous Physics Philosophy on the Concept of Ether Throughout the History of Science: Birth, Death and Revival, Foundations of Science, 18 (3), 2013, pp. 559-577.

Plato, Epinomis, London, The Loeb Classical Library, Harvard University Press/ William Heinemann, 1950.

Plato, Timaeus, London, The Loeb Classical Library, Harvard University Press/ William Heinemann, 1952.

Platón, Obras Completas, Madrid, Aguilar, 1972.

Plutarch, Plutarch's Morals, Cambridge, Press Of John Wilson and Son, 1874. Ed. William W. Goodwin.

Reale, Giovanni, Por una nueva interpretación de Platón, Barcelona, Herder, 2003 (1997).

Taylor, Alfred, A Commentary on Plato's Timaeus, Oxford, The Clarendon Press, 1928.

Van Der Waerden, B. L., The motion of Venus, Mercury and the Sun in early greek astronomy, Archive for History of Exact Science, 26 (2), 1982, pp. 99-113. Visintainer, John, A Potential Infinity of Triangle Types. On the Chemistry of Plato's Timaeus, International Journal for Philosophy of Chemistry, 4 (2), 1998, pp. 117-128.

Vlastos, Gregory, The role of observation in Plato's conception of astronomy. En Anton, J. P. (ed.), Science and the Sciences in Plato, Delmar-New York, Caravan Books, 1980.

Wildberg, C., John Philoponus Criticism of Aristotle's Theory of Aether, Berlin/ New York, de Gruyter, 1988.

Zeyl, Donald J., Timaeus, Indianapolis, Hackett Publishing Co., 2000.

Zhmud, Leonid, Plato as 'Architect of Science', Phronesis, 43 (3), 1998, pp. 211-244. 